\title{
GLOBAL EXISTENCE AND ASYMPTOTIC BEHAVIOR OF SOLUTION OF SECOND-ORDER NONLINEAR IMPULSIVE DIFFERENTIAL EQUATIONS
}

\author{
DENGHUA CHENG and JURANG YAN
}

(Received 26 August 1996)

\begin{abstract}
AвSTRACT. We consider the global existence and asymptotic behavior of solution of second-order nonlinear impulsive differential equations.
\end{abstract}

2000 Mathematics Subject Classification. Primary 39A10, 39A12.

1. Introduction. In recent years, there have been many papers considering the impulsive differential equations, see, for example, $[2,3,1,4,5]$ and the references cited in [3]. In this paper, our results extend those in [6]. We consider the second-order nonlinear impulsive differential equation

$$
\begin{aligned}
& \left(p(t) y^{\prime}(t)\right)^{\prime}=f\left(t, y(t), y^{\prime}(t)\right), \quad t \neq t_{k}, t \geq 0, \\
& y\left(t_{k}^{+}\right)-y\left(t_{k}\right)=I_{k}\left(y\left(t_{k}\right)\right), \\
& y^{\prime}\left(t_{k}^{+}\right)-y^{\prime}\left(t_{k}\right)=J_{k}\left(y^{\prime}\left(t_{k}\right)\right), \quad k=1,2, \ldots,
\end{aligned}
$$

under the following standing assumptions on $p, f, I_{k}$ and $J_{k}$ :

$\left(\mathrm{A}_{1}\right) p:[0, \infty) \rightarrow(0, \infty)$ is continuous and

$$
P(t)=\int_{0}^{t} \frac{1}{p(s)} d s \rightarrow \infty \quad \text { as } t \rightarrow \infty ;
$$

$\left(\mathrm{A}_{2}\right) f:[0, \infty) \times \mathbb{R} \times \mathbb{R} \rightarrow(0, \infty)$ is continuous and $f(t, u, v)$ is nondecreasing in $u$ and $v$;

$\left(\mathrm{A}_{3}\right) I_{k}$ and $J_{k}: \mathbb{R} \rightarrow(0, \infty)$ are continuous and $I_{k}$ and $J_{k}$ are nondecreasing for $k=1,2, \ldots$;

$\left(\mathrm{A}_{4}\right) 0 \leq t_{0}<t_{1}<t_{2}<\cdots<t_{n}<\cdots$ with $\lim _{n \rightarrow \infty} t_{n}=\infty$;

$\left(\mathrm{A}_{5}\right)$ Every Cauchy problem for (1.1), (1.2), and (1.3) has a unique solution.

Let $y(t)$ be a solution of (1.1), (1.2), and (1.3) with the maximal interval of existence $\left[0, T_{y}\right)$. From (1.1), (1.2), and (1.3) we have $\left(p(t) y^{\prime}(t)\right)^{\prime}>0$ for $t \neq t_{k}$, so that $p(t) y^{\prime}(t)$ is increasing on $\left[0, T_{y}\right)$. It happens that either $T_{y}<\infty$ and $\lim _{t \rightarrow T_{y}} p(t) y^{\prime}(t)=\infty$, or else $T_{y}=\infty$ and $\lim _{t \rightarrow \infty} p(t) y^{\prime}(t)$ exists in $\mathbb{R} \cup\{\infty\}$. In the former case $y(t)$ is called a singular solution, and in the latter $y(t)$ is called a proper solution. The set of proper solutions of (1.1), (1.2), and (1.3) is further classified into the following four classes:

(i) the class of strongly increasing solutions consisting of all solutions $y(t)$ such that $\lim _{t \rightarrow \infty} p(t) y^{\prime}(t)=\infty$;

(ii) the class of weakly increasing solutions consisting of all solutions $y(t)$ such that $\lim _{t \rightarrow \infty} p(t) y^{\prime}(t) \in(0, \infty)$; 
(iii) the class of weakly decreasing solutions consisting of all solutions $y(t)$ such that $\lim _{t \rightarrow \infty} p(t) y^{\prime}(t)=0$;

(iv) the class of strongly decreasing solutions consisting of all solutions $y(t)$ such that $\lim _{t \rightarrow \infty} p(t) y^{\prime}(t) \in(-\infty, 0)$.

The main objective of this paper is to give explicit sufficient conditions for existence of some or all of these classes of proper solutions of (1.1), (1.2), and (1.3) defined on the given interval $[0, \infty)$.

2. Main results. We begin by giving a condition under which (1.1), (1.2), and (1.3) have strongly decreasing solutions.

THEOREM 2.1. Suppose that there exist constants $c>0$ and $I_{0}>0$ such that

$$
\begin{gathered}
\int_{0}^{\infty} f\left(t,-c P(t),-\frac{c}{p(t)}\right) d t<\infty, \\
\sum_{k=1}^{\infty} I_{k}(\cdot)<I_{0}, \quad \sum_{k=1}^{\infty} p\left(t_{k}\right) J_{k}\left(-\frac{c}{p\left(t_{k}\right)}\right)<\infty .
\end{gathered}
$$

Then, for any $b \in(c, \infty)$ and $\gamma \in \mathbb{R},(1.1),(1.2)$, and (1.3) have a strongly decreasing solution $y(t)$ satisfying

$$
y(0)=\gamma, \quad \lim _{t \rightarrow \infty} p(t) y^{\prime}(t)=-b .
$$

Proof. From (2.1), $\left(\mathrm{A}_{2}\right)$, and $\left(\mathrm{A}_{3}\right)$, we have

$$
\int_{0}^{\infty} f\left(t, \gamma+I_{0}-b P(t),-\frac{b}{p(t)}\right) d t<\infty, \quad \sum_{k=1}^{\infty} p\left(t_{k}\right) J_{k}\left(-\frac{b}{p\left(t_{k}\right)}\right)<\infty .
$$

Let $\Omega$ denote the Frechet space of all functions $y(t):[0, \infty) \rightarrow \mathbb{R}$, such that $y(t)$ is twice continuously differentiable for $t \neq t_{k}, y\left(t^{-}\right), y\left(t^{+}\right), y^{\prime}\left(t^{-}\right), y^{\prime}\left(t^{+}\right)$exist and $y\left(t^{-}\right)=y(t), y^{\prime}\left(t^{-}\right)=y^{\prime}(t)$ at $t=t_{k}$, with the usual metric topology, and $M$ be the set of all $y(t) \in \Omega$ that satisfy the following inequalities:

$$
\begin{gathered}
\gamma-b P(t)-\int_{0}^{t} \frac{1}{p(s)} \int_{s}^{\infty} f\left(\tau, \gamma+I_{0}-b P(\tau),-\frac{b}{p(\tau)}\right) d \tau d s \\
-P(t) \sum_{t \leq t_{k}} p\left(t_{k}\right) J_{k}\left(-\frac{b}{p\left(t_{k}\right)}\right)-\sum_{t \leq t_{k}} I_{k}\left(\gamma+I_{0}-b P\left(t_{k}\right)\right) \leq y(t) \leq \gamma+I_{0}-b P(t), \\
-b-\int_{t}^{\infty} f\left(s, \gamma+I_{0}-b P(s),-\frac{b}{p(s)}\right) d s-\sum_{t \leq t_{k}} p\left(t_{k}\right) J_{k}\left(-\frac{b}{p\left(t_{k}\right)}\right) \leq p(t) y^{\prime}(t) \leq-b, \quad t \geq 0 .
\end{gathered}
$$

Clearly, $M$ is a nonempty closed convex subset of $\Omega$. Define the operator $U: M \rightarrow \Omega$ by

$$
\begin{aligned}
U y(t)= & \gamma-b P(t)-\int_{0}^{t} \frac{1}{p(s)} \int_{s}^{\infty} f\left(\tau, y(\tau), y^{\prime}(\tau)\right) d \tau d s \\
& -P(t) \sum_{t \leq t_{k}} p\left(t_{k}\right) J_{k}\left(y^{\prime}\left(t_{k}\right)\right)+\sum_{t_{k}<t} I_{k}\left(y\left(t_{k}\right)\right), \quad t \geq 0 .
\end{aligned}
$$


It is easy to verify that $U M \subset M, U$ is continuous and $\overline{U M}$ is compact. So, the SchauderTychonoff fixed point theorem implies that $U$ has a fixed point $y$ in $M$. This fixed point $y(t)$ is a strongly decreasing solution of (1.1), (1.2), and (1.3) satisfying (2.2). This completes the proof.

EXAMPLE 2.2. Consider the equation

$$
\begin{aligned}
& y^{\prime \prime}=a(t) e^{y^{\prime}}, \quad t \neq t_{k}, t \geq 0, \\
& y\left(t_{k}^{+}\right)-y\left(t_{k}\right)=m_{k}\left(\frac{\pi}{2}+\arctan y\left(t_{k}\right)\right), \\
& y^{\prime}\left(t_{k}^{+}\right)-y^{\prime}\left(t_{k}\right)=\ln \left(1+M_{k} e^{y^{\prime}\left(t_{k}\right)}\right), \quad k=1,2, \ldots
\end{aligned}
$$

If $\int_{0}^{\infty} a(t) d t<\infty$, and $\sum_{k=1}^{\infty} m_{k}<\infty$ and $\sum_{k=1}^{\infty} M_{k}<\infty$, then for any $b \in(c, \infty)$ and $\gamma \in \mathbb{R}$, (2.6) has a strongly decreasing solution

$$
y(t)=\gamma-\int_{0}^{t} \ln \left[e^{b}+\int_{s}^{\infty} a(u) d u+\sum_{t_{k} \geq s} \frac{M_{k}}{1+M_{k} e^{y^{\prime}\left(t_{k}\right)}}\right] d s+\sum_{t_{k}<t} m_{k}\left(\frac{\pi}{2}+\arctan y\left(t_{k}\right)\right)
$$

satisfying $y(0)=\gamma$ and $\lim _{t \rightarrow \infty} y^{\prime}(t)=-b$.

We now give a simple lemma which will be useful in the following discussions, and the proof of the lemma is straightforward by induction and will be omitted.

LEMMA 2.3. Together with (1.1), (1.2), and (1.3) we consider the equation

$$
\begin{aligned}
& \left(p(t) z^{\prime}(t)\right)^{\prime}=g\left(t, z(t), z^{\prime}(t)\right), \quad t \neq t_{k}, t \geq 0, \\
& z\left(t_{k}^{+}\right)-z\left(t_{k}\right)=I_{k}^{*}\left(z\left(t_{k}\right)\right), \\
& z^{\prime}\left(t_{k}^{+}\right)-z^{\prime}\left(t_{k}\right)=J_{k}^{*}\left(z^{\prime}\left(t_{k}\right)\right), \quad k=1,2, \ldots,
\end{aligned}
$$

where $p(t)$ is as in (1.1), $g:[0, \infty) \times \mathbb{R} \times \mathbb{R} \rightarrow(0, \infty)$ is continuous and nondecreasing in the last two variables, $I_{k}^{*}, J_{k}^{*}$ are also continuous and nondecreasing from $\mathbb{R}$ to $(0, \infty)$, and

$$
\begin{aligned}
f(t, u, v) & \geq g(t, u, v), \quad(t, u, v) \in[0, \infty) \times \mathbb{R} \times \mathbb{R}, \\
I_{k}\left(u\left(t_{k}\right)\right) & \geq I_{k}^{*}\left(u\left(t_{k}\right)\right), \\
J_{k}\left(u^{\prime}\left(t_{k}\right)\right) & \geq J_{k}^{*}\left(u^{\prime}\left(t_{k}\right)\right), \quad k=1,2, \ldots
\end{aligned}
$$

Let $y(t)$ and $z(t)$ be solutions of (1.1), (1.2), (1.3), and (2.8), respectively, satisfying $z\left(a^{+}\right) \leq y\left(a^{+}\right)$and $z^{\prime}\left(a^{+}\right)<y^{\prime}\left(a^{+}\right)$. If $y(t)$ is defined on $[a, b)$, then $z(t)$ exists on $[a, b)$ and satisfies $z(t)<y(t)$ and $z^{\prime}(t)<y^{\prime}(t)$ for $t \in(a, b)$.

THEOREM 2.4. Suppose that (2.1) hold for all $c>0$. Then for any $\gamma \in \mathbb{R},(1.1),(1.2)$, and (1.3) have a unique weakly decreasing solution $y(t)$ satisfying $y(0)=\gamma$.

Proof. We fix $\gamma \in \mathbb{R}$. Let $y_{\alpha}(t)$ denote the solution of (1.1), (1.2), and (1.3) satisfying $y(0)=\gamma$ and $p(0) y^{\prime}(0)=\alpha$. We define the set $A \subset \mathbb{R}$ by

$$
A=\left\{\alpha \in \mathbb{R}: y_{\alpha}(t) \text { is a strongly decreasing solution }\right\},
$$


which is nonempty by Theorem 2.1. Now we show that $A$ is an open set which is bounded above. Let $\alpha \in A$. If $\beta<\alpha$, then by Lemma $2.3\left(g \equiv f, I_{k}^{*} \equiv I_{k}\right.$ and $J_{k}^{*} \equiv J_{k}$ ), $y_{\beta}(t)$ is a strongly decreasing solution, that is, $\beta \in A$. Suppose that $\beta>\alpha$. Since $\alpha \in A$, there exists an $l>0$ such that $\lim _{n \rightarrow \infty} p(t) y_{\alpha}^{\prime}=-l$. We choose $t_{l}>0$ large enough so that

$$
\int_{t_{l}}^{\infty} f\left(t, \gamma+I_{0}-\frac{l}{2} P(t),-\frac{l}{2 p(t)}\right) d t<\frac{l}{4}, \quad \sum_{t_{k}>t_{l}} p\left(t_{k}\right) J_{k}\left(-\frac{l}{2 p\left(t_{k}\right)}\right)<\frac{l}{4} .
$$

By the continuous dependence on initial conditions, for all $\beta>\alpha$ sufficiently close to $\alpha, y_{\beta}(t)$ exist on $\left[0, t_{l}\right]$ and satisfy $p(t) y_{\beta}^{\prime}(t)<-l$ for $t \in\left[0, t_{l}\right]$. It can be shown that for such a $\beta>\alpha, y_{\beta}(t)$ can be extended to $[0, \infty)$, and satisfies

$$
p(t) y_{\beta}^{\prime}(t)<-\frac{l}{2} \text { for } t \geq 0 .
$$

In fact, if (2.12) fails, then there exists $t_{m}>t_{1}$ such that

$$
p\left(t_{m}\right) y_{\beta}^{\prime}\left(t_{m}\right)=-\frac{l}{2}, \quad p(t) y_{\beta}^{\prime}(t)<-\frac{l}{2}
$$

for $t \in\left[0, t_{m}\right)$ and $t \neq t_{k}, t_{k} \in\left[0, t_{m}\right)$. Integrating (1.1) and using (2.11), (2.12), and (2.13), we have

$$
\begin{aligned}
-\frac{l}{2} & =p\left(t_{m}\right) y_{\beta}^{\prime}\left(t_{m}\right)=p\left(t_{l}\right) y_{\beta}^{\prime}\left(t_{l}\right)+\int_{t_{l}}^{t_{m}} f\left(t, y(t), y^{\prime}(t)\right) d t+\sum_{t_{l} \leq t_{k}<t_{m}} p\left(t_{k}\right) J_{k}\left(y\left(t_{k}\right)\right) \\
& \leq-l+\int_{t_{l}}^{t_{m}} f\left(t, \gamma+I_{0}-\frac{l}{2} P(t),-\frac{l}{2 p(t)}\right) d t+\sum_{t_{l} \leq t_{k}<t_{m}} p\left(t_{k}\right) J_{k}\left(-\frac{l}{2 p\left(t_{k}\right)}\right) \\
& \leq-l+\int_{t_{l}}^{\infty} f\left(t, \gamma+I_{0}-\frac{l}{2} P(t),-\frac{l}{2 p(t)}\right) d t+\sum_{t_{l} \leq t_{k}} p\left(t_{k}\right) J_{k}\left(-\frac{l}{2 p\left(t_{k}\right)}\right) \\
& <-l+\frac{l}{4}+\frac{l}{4}=-\frac{l}{2} .
\end{aligned}
$$

This contradiction proves that (2.12) holds, and this implies $\beta \in A$. Thus $A$ is open. On the other hand, if $\alpha \geq 0$, then $\alpha \notin A$, so that $A$ is bounded from above, we put $\alpha^{*}=\sup A$. It is obvious that $\alpha^{*} \notin A$ and $\alpha^{*} \leq 0$.

We consider the solution $y_{\alpha^{*}}(t)$. By the continuous dependence on the initial conditions, $y_{\alpha^{*}}(t)$ is not a singular solution, that is $y_{\alpha^{*}}(t)$ exists on $[0, \infty)$ and satisfies $\lim _{n \rightarrow \infty} p(t) y_{\alpha^{*}}^{\prime}(t)=\eta^{*} \geq 0\left(\eta^{*}\right.$ may be $\left.\infty\right)$. The continuous dependence on initial conditions precludes the possibility that $\eta^{*}$ is positive, and so we must have $\eta^{*}=0$. This means that $y_{\alpha^{*}}$ is a weakly decreasing solution passing through $(0, \gamma)$.

To prove the uniqueness of the weakly decreasing solution passing through $(0, \gamma)$, let $y_{1}(t)$ and $y_{2}(t)$ be two weakly decreasing solutions of (1.1), (1.2), and (1.3) such that $y_{1}(0)=y_{2}(0)=\gamma$ but $y_{1}^{\prime}(0)<y_{2}^{\prime}(0)$. Lemma $2.3(g \equiv f), I_{k}^{*} \equiv I_{k}$ and $J_{k}^{*} \equiv J_{k}$ implies that $y_{1}(t) \leq y_{2}(t)$ and $y_{1}^{\prime}(t) \leq y_{2}^{\prime}(t)$ for $t \geq 0$. It follows from (1.1) that $\left[p(t)\left(y_{2}^{\prime}(t)-y_{1}^{\prime}(t)\right)\right]^{\prime}=f\left(t, y_{2}, y_{2}^{\prime}\right)-f\left(t, y_{1}, y_{1}^{\prime}\right) \geq 0$ for $t \geq 0, t \neq t_{k}$. So $p(t) y_{2}^{\prime}(t)-$ $p(t) y_{1}^{\prime}(t) \geq p(0)\left[y_{2}^{\prime}(0)-y_{1}^{\prime}(0)\right]>0$ for $t \geq 0$. Since the left-hand side of this inequality tends to 0 as $t \rightarrow \infty$, we have a contradiction. This completes the proof. 
The following theorem gives a useful information about the asymptotic behavior of weakly decreasing solutions of (1.1), (1.2), and (1.3).

THEOREM 2.5. All weakly decreasing solutions of (1.1), (1.2), and (1.3), if any, are either simultaneously bounded or simultaneously unbounded.

Proof. Let $y_{1}(t)$ and $y_{2}(t)$ be the weakly decreasing solutions satisfying $y_{1}(0)=$ $\gamma_{1}$ and $y_{2}(0)=\gamma_{2}$ with $\gamma_{1}<\gamma_{2}$. It suffices to prove that the difference $y_{2}(t)-y_{1}(t)$ is a positive nonincreasing function on $[0, \infty)$. First we show that $y_{2}(t)>y_{1}(t)$ for $t \geq 0$. Otherwise, there exists $t^{*}>0$ such that $y_{1}\left(t^{*}\right)=y_{2}\left(t^{*}\right)$ and $y_{1}^{\prime}\left(t^{*}\right)>y_{2}^{\prime}\left(t^{*}\right)$. We choose $t^{* *}>t^{*}$ sufficiently close to $t^{*}$, such that $y_{1}\left(t^{* *}\right)>y_{2}\left(t^{* *}\right)$ and fix it. By the continuous dependence on initial data, a solution $\tilde{y}(t)$ of (1.1), (1.2), and (1.3) with $\tilde{y}(0)=\gamma_{2}$ satisfies $y_{2}\left(t^{* *}\right)<\tilde{y}\left(t^{* *}\right)<y_{1}\left(t^{* *}\right)$ and $y_{2}^{\prime}\left(t^{* *}\right)<\tilde{y}^{\prime}\left(t^{* *}\right)<y_{1}^{\prime}\left(t^{* *}\right)$, provided $\tilde{y}^{\prime}(0)-y_{2}^{\prime}(0)>0$ is sufficiently small. By Lemma $2.3, \tilde{y}(t)$ exists on $[0, \infty)$ and satisfies $y_{2}^{\prime}(t)<\tilde{y}^{\prime}(t)<y_{1}^{\prime}(t)$, for $t \geq t^{* *}$, that is, $p(t) y_{2}^{\prime}(t)<p(t) \tilde{y}^{\prime}(t)<$ $p(t) y_{1}^{\prime}(t)$ for $t \geq t^{* *}$. This fact means that $\tilde{y}$ is a weakly decreasing solution passing through $\left(0, \gamma_{2}\right)$, which contradicts the uniqueness of the weakly decreasing solution passing through $\left(0, \gamma_{2}\right)$. Thus we obtain $y_{2}(t)>y_{1}(t)$ for $t \geq 0$. Next, if there exists $\tau \geq 0$ such that $y_{1}^{\prime}(\tau)<y_{2}^{\prime}(\tau)$, then the same argument as above leads us to the conclusion that there is a weakly decreasing solution different from $y_{2}(t)$ passing through $\left(0, \gamma_{2}\right)$. This again is a contradiction, and so we have $y_{2}^{\prime}(t) \leq y_{1}^{\prime}(t)$ for $t \geq 0$. It follows that $y_{2}(t)-y_{1}(t)$ is a positive nonincreasing function for $t \geq 0$, and the proof is complete.

We now obtain conditions guaranteeing the existence of singular solutions of (1.1), (1.2), and (1.3).

THEOREM 2.6. Suppose $\sum_{k=1}^{\infty} I_{k}(\cdot)<I_{0}$ and that there exists a positive continuous function $f_{*}(t, u, v)$ on $[0, \infty) \times \mathbb{R} \times \mathbb{R}$ which is nonincreasing in $t$ and nondecreasing in $u$ and $v$, and satisfies $f(t, u, v) \geq f_{*}(t, u, v)$ on $[0, \infty) \times \mathbb{R} \times \mathbb{R}$. Moreover suppose that $p(t) P(t)$ is nondecreasing. We define

$$
F_{\gamma}(t, u)=\int_{\gamma}^{u} f_{*}\left(t, s, \frac{s-\gamma-I_{0}}{p(t) P(t)}\right) d s \quad \text { for } \gamma \in \mathbb{R}, t>0, u>\gamma .
$$

If

$$
\int^{\infty}\left(F_{\gamma}(t, u)\right)^{-1 / 2} d u<\infty \text { for any } t>0,
$$

then for every $t_{0} \geq 0$, there exists a singular solution $y(t)$ of (1.1), (1.2), and (1.3) satisfying $y\left(t_{0}\right)=\gamma$.

Proof. We fix $t^{*}>t_{0} \geq 0$. Let $m$ and $M$ be positive constants such that $m \leq p(t) \leq$ $M$ for $t \in\left[t_{0}, t^{*}\right]$. Choose $\delta=\delta\left(\gamma, t_{0}\right)>0$ large enough so that

$$
M \int_{\gamma}^{+\infty}\left(2 m F_{\gamma}\left(t^{*}, u\right)+\delta^{2}\right)^{-1 / 2} d u<t^{*}-t_{0} .
$$

Now we show that the solution $y(t)$ of (1.1), (1.2), and (1.3) satisfying the initial conditions $y\left(t_{0}\right)=\gamma$ and $p\left(t_{0}\right) y^{\prime}\left(t_{0}\right) \geq \delta$ cannot exist on $\left[t_{0}, t^{*}\right]$. Suppose the contrary, 
then from (1.1) and the monotonicity of $p(t) y^{\prime}(t)$, we see that

$$
\left(\left(p(t) y^{\prime}(t)\right)^{2}\right)^{\prime} \geq 2 p(t) y^{\prime}(t) f_{*}\left(t, y(t), y^{\prime}(t)\right), \quad t \in\left[t_{0}, t^{*}\right], t \neq t_{k} \in\left[t_{0}, t^{*}\right] .
$$

On the other hand, we have

$$
\begin{aligned}
y(t) & =\int_{t_{0}}^{t} y^{\prime}(s) d s+\gamma+\sum_{t_{k}<t} I_{k}\left(y\left(t_{k}\right)\right) \\
& =\int_{t_{0}}^{t} \frac{p(s) y^{\prime}(s)}{p(s)} d s+\gamma+\sum_{t_{k}<t} I_{k}\left(y\left(t_{k}\right)\right) \\
& \leq p(t) y^{\prime}(t) \int_{t_{0}}^{t} \frac{1}{p(s)} d s+\gamma+\sum_{k=1}^{\infty} I_{k}\left(y\left(t_{k}\right)\right) \\
& \leq p(t) P(t) y^{\prime}(t)+\gamma+I_{0}, \quad t \in\left[t_{0}, t^{*}\right],
\end{aligned}
$$

that is,

$$
y^{\prime}(t) \geq \frac{y(t)-\gamma-I_{0}}{p(t) P(t)}, \quad t \in\left[t_{0}, t^{*}\right] .
$$

Integrating (2.18) from $t_{0}$ to $t \in\left[t_{0}, t^{*}\right]$ and using (2.20) and the monotonicity condition imposed on $f_{*}$, we obtain

$$
\begin{aligned}
\left(p(t) y^{\prime}(t)\right)^{2} \geq & 2 m \int_{t_{0}}^{t} y^{\prime}(s) f_{*}\left(s, y(s), \frac{y(s)-\gamma-I_{0}}{p(s) P(s)}\right) d s+\delta^{2} \\
& +\sum_{t_{k}<t} p^{2}\left(t_{k}\right)\left[2 y^{\prime}\left(t_{k}\right) J_{k}\left(y^{\prime}\left(t_{k}\right)\right)+J_{k}^{2}\left(y^{\prime}\left(t_{k}\right)\right)\right] \\
\geq & 2 m \int_{t_{0}}^{t} y^{\prime}(s) f_{*}\left(t, y(s), \frac{y(s)-\gamma-I_{0}}{p(t) P(t)}\right) d s+\delta^{2}
\end{aligned}
$$

for $t \in\left[t_{0}, t^{*}\right]$, which is equivalent to

$$
M y^{\prime}(t) \geq\left(2 m \int_{\gamma}^{y(t)} f_{*}\left(t, s, \frac{s-\gamma-I_{0}}{p(t) P(t)}\right) d s+\delta^{2}\right)^{1 / 2}, \quad t \in\left[t_{0}, t^{*}\right]
$$

In view of the monotonicity of $f_{*}$, this implies

$$
M y^{\prime}(t)\left(2 m F_{\gamma}\left(t^{*}, y(t)\right)+\delta^{2}\right)^{-1 / 2} \geq 1, \quad t \in\left[t_{0}, t^{*}\right]
$$

Integrating from $t_{0}$ to $t^{*}$, and using (2.17) we obtain

$$
\begin{aligned}
t^{*}-t_{0} & >M \int_{\gamma}^{\infty}\left(2 m F_{\gamma}\left(t^{*}, u\right)+\delta^{2}\right)^{-1 / 2} d u \\
& \geq M \int_{\gamma}^{y\left(t^{*}\right)}\left(2 m F_{\gamma}\left(t^{*}, u\right)+\delta^{2}\right)^{-1 / 2} d u \geq t^{*}-t_{0},
\end{aligned}
$$

which is a contradiction. Thus this solution $y(t)$ must be singular. 
We now turn to the problem of finding conditions for (1.1), (1.2), and (1.3) to have weakly and strongly increasing solutions.

THEOREM 2.7. Suppose that there exist constants $c>0$ and $I_{0}>0$ such that

$$
\begin{gathered}
\int_{0}^{\infty} f\left(t, c P(t), \frac{c}{p(t)}\right) d t<\infty, \\
\sum_{k=1}^{\infty} I_{k}(\cdot)<I_{0}, \quad \sum_{k=1}^{\infty} p\left(t_{k}\right) J_{k}\left(\frac{c}{p\left(t_{k}\right)}\right)<\infty .
\end{gathered}
$$

Then for any $b \in(0, c)$ and any $\gamma \in \mathbb{R}$, equations (1.1), (1.2), and (1.3) have a weakly increasing solution $y(t)$ satisfying

$$
y(0)=\gamma, \quad \lim _{t \rightarrow \infty} p(t) y^{\prime}(t)=b .
$$

Proof. We omit the proof as it is virtually the same as that of Theorem 2.1.

THEOREM 2.8. Suppose that the assumptions of Theorem 2.6 are satisfied for any $\gamma \in \mathbb{R}$. If (2.25) hold for all $c>0$, then for any $\gamma \in \mathbb{R},(1.1),(1.2)$, and (1.3) have a strongly increasing solution $y(t)$ satisfying $y(0)=\gamma$.

Proof. Let $\gamma \in \mathbb{R}$ be fixed and let $y_{\alpha}(t)$ be the solution of (1.1), (1.2), and (1.3) satisfying $y(0)=\gamma$ and $p(0) y^{\prime}(0)=\alpha$. We define the sets $A, B \subset \mathbb{R}$ by

$$
\begin{aligned}
& A=\left\{\alpha \in \mathbb{R}: y_{\alpha}(t) \text { is a weakly increasing solution }\right\} \\
& B=\left\{\alpha \in \mathbb{R}: y_{\alpha}(t) \text { is a singular solution }\right\} .
\end{aligned}
$$

By Theorems 2.6 and 2.7, we see that $B \neq \varnothing$ and $A \neq \varnothing$. Lemma 2.3 implies that $\alpha \leq \beta$ for any $\alpha \in A$ and $\beta \in B$. Similar to the proof of Theorem 2.4 we can show that $A$ and $B$ are disjoint open subsets of $\mathbb{R}$. We put $\alpha^{*}=\sup A$ and $\beta_{*}=\inf B$. It is easily seen that $\alpha^{*} \notin A, \beta_{*} \notin B$, and $\alpha^{*} \leq \beta_{*}$. Then, for any $\alpha \in\left[\alpha^{*}, \beta_{*}\right]$ (which may be reduced to one point), $y_{\alpha}(t)$ is a strongly increasing solution of (1.1), (1.2), and (1.3) satisfying $y(0)=\gamma$. This completes the proof.

\section{REFERENCES}

[1] A. B. Dishliev and D. D. Baĭnov, Continuous dependence of the solution of a system of differential equations with impulses on the initial condition, Comment. Math. Prace Mat. 30 (1990), no. 1, 51-67 (1991). MR 92m:34027. Zbl 744.34016.

[2] G. S. Ladde, V. Lakshmikantham, and B. G. Zhang, Oscillation Theory of Differential Equations with Deviating Arguments, Monographs and Textbooks in Pure and Applied Mathematics, vol. 110, Marcel Dekker, Inc., New York, 1987. MR 90h:34118. Zbl 832.34071.

[3] V. Lakshmikantham, D. D. Bănov, and P. S. Simeonov, Theory of Impulsive Differential Equations, Series in Modern Applied Mathematics, vol. 6, World Scientific Publishing Co., Inc., Teaneck, NJ, 1989. MR 91m:34013. Zbl 719.34002.

[4] P. S. Simeonov and D. D. Bainnov, On the asymptotic equivalence of systems with impulse effect, J. Math. Anal. Appl. 135 (1988), no. 2, 591-610. MR 89m:34078. Zbl 671.34051.

[5] _ On the asymptotic properties of the solutions of systems of differential equations with impulse effect, J. Math. Anal. Appl. 158 (1991), no. 1, 1-14. MR 92m:34028. Zbl 739.34019. 
[6] H. Usami, Global existence and asymptotic behavior of solutions of second-order nonlinear differential equations, J. Math. Anal. Appl. 122 (1987), no. 1, 152-171. MR 88c:34044. Zbl 644.34011.

Denghua Cheng: TAIYuan TV University, TAIyuan, ShanXi 030001, China

E-mail address: tyddcdh@emai 1.com.cn

JURANG YAN: DePaRTMENT OF MATHEMATICS, ShanXi UniVERSITY, TAIYUAN, ShanXi 030006, CHINA

E-mail address: jeyan@sxu.edu.cn 


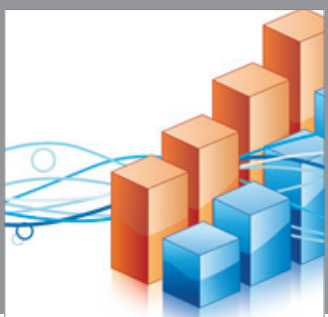

Advances in

Operations Research

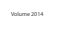

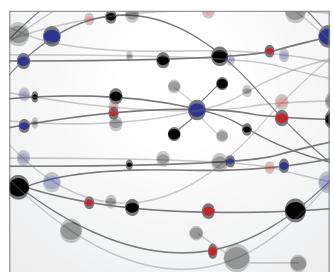

\section{The Scientific} World Journal
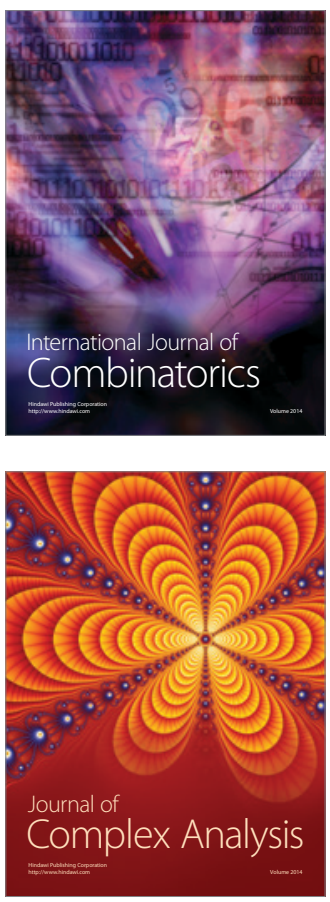

International Journal of

Mathematics and

Mathematical

Sciences
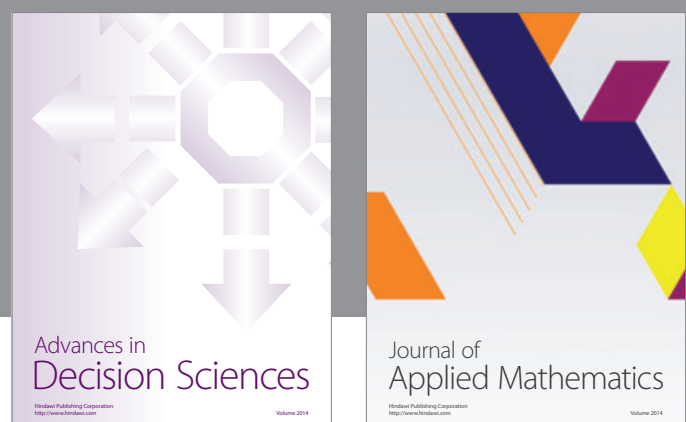

Journal of

Applied Mathematics
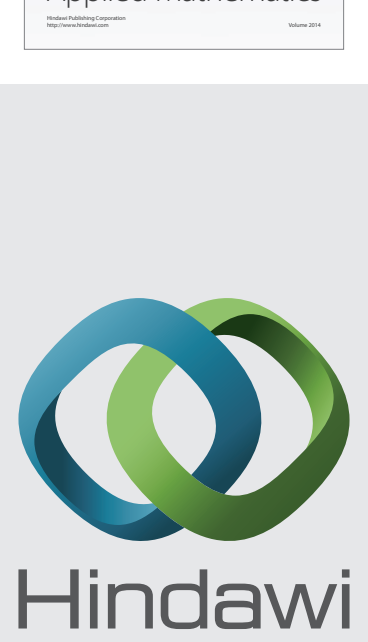

Submit your manuscripts at http://www.hindawi.com
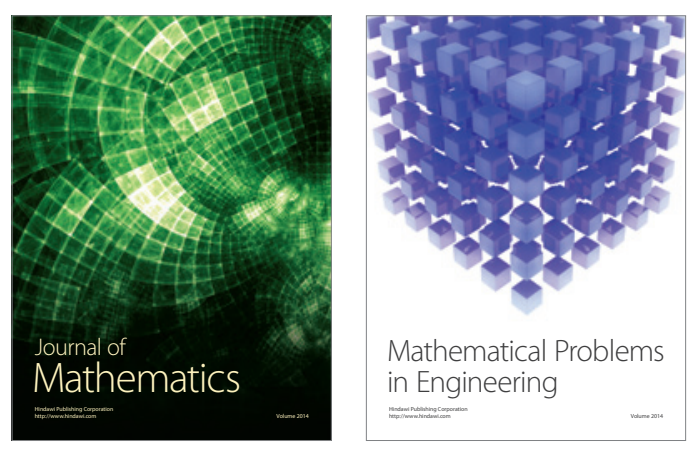

Mathematical Problems in Engineering
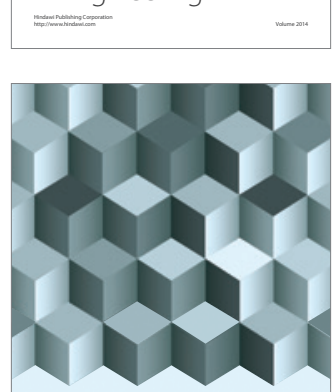

Journal of

Function Spaces
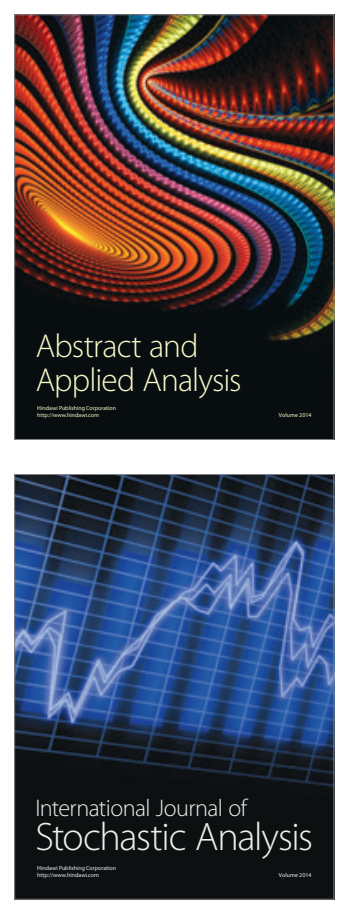

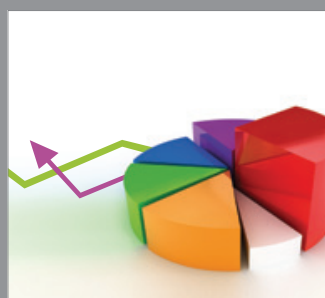

ournal of

Probability and Statistics

Promensencen
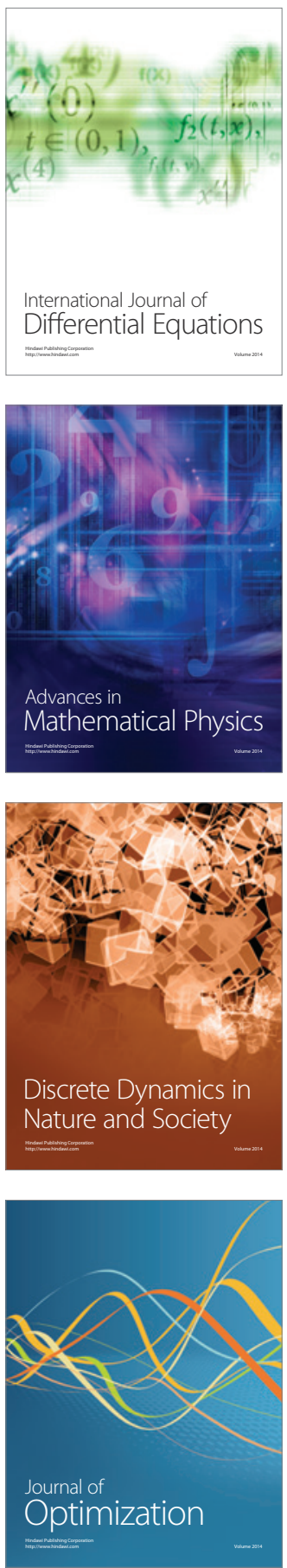